\title{
SEVERE LEPTOSPIROSIS - A CASE SERIES AND REVIEW
}

\author{
*B.W.P Habaragamuwa ${ }^{1}$ Gihan Piyasiri', \\ Senior Registrar Anaesthesia and Critical Care, Colombo South Teaching Hospital, Kalubowila ${ }^{1}$, \\ Registrar Anaesthesia, Colombo South Teaching Hospital, Kalubowila ${ }^{2}$.
}

*Corresponding author: budhi190@yahoo.com

\section{Background}

Key words: organ, score, system

\begin{abstract}
The purpose of this retrospective case review was to identify different patterns of clinical presentation of severe leptospirosis, and to find out any correlation between organ specific complication/s and outcome of the patients.
\end{abstract}

\section{Method}

Leptospirosis patients who were admitted to the intensive care unit (ICU) at the Colombo South Teaching Hospital during August to September 2008 were included. Organ specific involvement in six organs (heart, lung, kidney, liver, vascular, brain and spinal cord) was recorded. One score was given to each organ involvement. Score was recorded on admission and during ICU stay. Outcome of the patient was recorded as either discharged or died in the ICU.

Results

Renal, hepatic and haematological involvement was found in all patients. 13(65\%) patients died and $7(35 \%)$ patients were discharged to the ward. All patients with four organ involvement were survived. All patients with six organ involvement died in the intensive care unit. 8(80\%) patients died and 3(20\%) patients survived among patients with five organ involvement.

Four or more organs were affected on admission to ICU in patients who died and only three organs were affected in discharged patients.

\section{Conclusion}

Outcome of severe leptospirosis was not determined by the involvement of renal, hepatic and haematological systems. Five or more organ involvement was associated with increased mortality. Combination of cardiac and respiratory system involvement or involvement of central nervous system was associated with bad outcome. Seeking critical care management at three organ involvement stage would improve the outcome of the patients.

Leptospirosis, an infectious disease that affects humans and animals, is considered the most common zoonosis in the world. ${ }^{1}$ The disease was first recognized as an occupational disease of sewer workers in 1883. In 1886, Weil described the clinical manifestations in four men who had severe jaundice, fever and haemorrhage with renal involvement.

Leptospirosis is caused by pathogenic spiral bacteria that belong to the genus leptospira, the family Leptospiraceae, and the order Spirochaetales.

According to the epidemiology report, there were 34 hospital reported deaths and 2175 cases of leptospirosis recorded in 2007. There were 41 hospital deaths reported by end of April 2008. The most vulnerable groups to leptospirosis in Sri Lanka are farmers, fresh water fishermen, gem miners and those cleaning canals and drains.

- To identify different patterns of clinical presentation of severe leptospirosis.

- To find out any correlation between organ specific involvement and outcome of the patients in order to identify severe leptospirosis early and to provide critical care management and/ or critical care outreach management.

\section{Materials and Methods}

We undertook a retrospective case review of leptospirosis patients who were admitted to the 
intensive care unit (ICU) at the Colombo South Teaching Hospital during August and September 2008.

Inclusion criteria: All patients who were suspected of having leptospirosis on clinical grounds and/or investigations including antibody levels.

Data were recorded from ICU monitoring records. Organ specific involvement was recorded on admission and during ICU stay. One score was given to the involvement of each organ. Score on admission to the ICU and highest score in the ICU stay were recorded. Following features used to identify the organ involvement and presence of at least one feature indicated the involvement of the organ.

- $\quad$ Respiratory $-\mathrm{PaO}_{2}: \mathrm{FiO}_{2}<300$, abnormal chest radiography.

- Cardiac - Systolic blood pressure $<90 \mathrm{mmHg}$, requirement of inotropic/vasopressor agent, arrhythmia, 2D echo cardiographic evidence of myocarditis.

- Renal - Oligouria, anuria, serum creatinine $>110 \mu \mathrm{mol} / \mathrm{l}$.

- Hepatic - icterus, hepatomegaly, serum bilirubin $>20 \mu \mathrm{mol} / 1$.

- Nervous system - Seizures, Glasgow coma score $<15 / 15$.

- Haematological - Bleeding manifestations, platelets $<100,000 / \mathrm{mm}^{3}$, INR $>1.5$.

Outcome was measured as either discharged to the ward or died in the ICU.

Confidentiality of the patients' information was strictly maintained and patients' management was not affected by the study.

\section{Results}

Twenty cases of leptospirosis patients were admitted to the ICU during the period. 16(80\%) were $\leq 60$ years and $4(20 \%)$ were $>60$ years. $18(90 \%)$ patients were males and $19(95 \%)$ had exposure to paddy fields.

Thrombocytopenia $\left(<100,000 / \mathrm{mm}^{3}\right)$ and some bleeding manifestations were recorded in all patients. Prolonged INR was found in only two patients. Spontaneous bleeding was observed even with a platelet count of $>20,000 / \mathrm{mm}^{3}$.

Hepatic involvement was found in all patients and transaminase levels never exceeded above 500U/1 in any of the patients. SGOT level was higher than SGPT level in all cases.

All except one case had evidence of renal impairment which was found on admission. 19(95\%) patients had kidney failure according to RIFLE classification. Peritoneal dialysis (PD) was used as a method of renal replacement. The average period of peritoneal dialysis in discharged patients was 9 days. PD catheter related infection was not encountered in any of the patients. Hyperkalemia was encountered only in one patient and it was very resistant to standard treatment. Serum potassium was low or normal in all other patients.

Hypotension and /or arrhythmias were noted in $18(90 \%)$ patients. Dobutamine was used as the first line inotropic agent and average period of inotropic/vasopressor treatment was 6 days in discharged patients.

Ventilator support was indicated in 16(80\%) patients and all had chest radiographic appearance of diffuse, ill defined, ground glass pattern and $\mathrm{PaO}_{2}: \mathrm{FiO}_{2}<300$. Adult respiratory distress syndrome or pulmonary haemorrhage could have resulted in the above features. One patient was intubated due to low Glasgow coma score.

Central nervous system (CNS) was affected in $6(30 \%)$ patients. All patients got generalized tonic- clonic seizures which were resistant to standard treatment. Thiopentone sodium infusion 10-40mg per hour was started to control seizures in these patients. CT brain showed no haemorrhages.

$13(65 \%)$ patients died and 7(35\%0 patients were discharged to the ward. Four or more organs were affected on admission to the ICU in patients who died in the ICU. This is as opposed to the fact that only 3 organs were affected in patients who were discharged to the ward.

All patients had four or more organ involvement during the ICU stay. Four organs (except nervous system) were involved in 5(25\%) patients and all were discharged to the ward. Five organs (except nervous system) were involved in $10(50 \%)$ patients and $7(70 \%)$ patients died and only three(30\%) patients were discharged. Six organs were 
involved in 6(30\%) patients and all died in the ICU.

All patents were treated with intravenous penicillin 1 megaunit 6 hourly and intravenous cefotaxime $1 \mathrm{~g}$ daily.

\section{Discussion}

According to this case series, $95 \%$ of patients had a preceding history of exposure to paddy fields which indicates the vulnerability of farmers to the disease.

Bleeding manifestations were found even with the platelet count of $>20,000 / \mathrm{mm}^{3}$ probably due to thrombasthenia associated with the illness. In this case series, we have transfused platelets to keep the levels at least $>50,000 / \mathrm{mm}^{3}$.

All patients except one were clinically icteric in this series. Jaundice indicates severe form of the disease, otherwise known as Weil disease. ${ }^{2}$ Hepatic necrosis is one of the features in Weil's syndrome. Modest rise in hepatic transaminase levels $(<500 \mathrm{IU} / \mathrm{l})$ was noted in this review and aspartate transaminase was higher than alanine transaminase in all patients. Prolonged INR was encountered in only two patients despite bleeding manifestations.

95\% of patients had kidney failure according to the RIFLE classification. Complete recovery of renal function (according to the serum creatinine) was evident in patients who were discharged to the ward. Hyperkalemia was an uncommon finding in this case series and it was found in only one patient.

Congestive cardiac failure, myocarditis and pericarditis may occur in severe leptospirosis. ${ }^{2}$ Cardiac involvement was noted in $90 \%$ of patients and it was evident by either hypotension and/or arrhythmia.

Pulmonary manifestations, both diffuse, ill defined, ground glass pattern in chest radiograph and $<200 \mathrm{PaO}_{2}: \mathrm{FiO}_{2}$ ratio, were found in $80 \%$ of patients. Endotracheal bleeding suggestive of pulmonary haemorrhage was also noted in all of these patients. Pulmonary involvement is the main cause of death due to leptospirosis in some countries, usually as a result of pulmonary haemorrhage or adult respiratory distress syndrome (ARDS). ${ }^{2,3}$ Indeed, the severe pulmonary form of leptospirosis (SPFL) is

considered to be one of the major causes of death in patients with severe leptospirosis. ${ }^{2}$ Yeon and colleague described three radiographic pattern of pulmonary involvement in leptospirosis patients: small nodular pattern, large confluent area of consolidation and diffuse, ill defined, ground glass pattern. They also noted that abnormalities were bilateral, non lobar in all cases and had a marked tendency towards peripheral predominance. ${ }^{4}$ Diffuse, ill defined, ground glass pattern was found in patients who had pulmonary involvement in this case series. Among the patients who got four organ involvements, two patients had pulmonary involvement as the fourth organ. These two patients survived and the average period of ventilation was 7 days. We practiced pressure controlled, low tidal volume $(5-7 \mathrm{ml} / \mathrm{kg})$, high respiratory rate (14-20 breath per minute), high Peak end expiratory pressure(PEEP) in all patients who were ventilated due to pulmonary involvement.

Meningeal symptoms developed in nearly $50 \%$ of patients in leptospirosis. Cranial nerve palsies, encephalitis and change in the level of consciousness are less common. ${ }^{2}$ Impaired level of consciousness and generalized tonic-clonic seizures were encountered in this case series when central nervous system was involved. Six patients got CNS involvement and all died. Generalized seizures, were very resistant to standard treatment, probably due to meningitis or encephalitis as CT brain showed no haemorrhages.

The highest mortality rates are in elderly patients and in those with Weil's syndrome. ${ }^{2}$ Average age among the dead patients was 54 years in this case series.

Involvement of renal, hepatic and haematological systems did not determine the outcome of patients in this case series as all three organs were affected in all patients. Patients who got four organ involvement (cardiac or respiratory as the fourth organ) were discharged to the ward. $80 \%$ of patients' with five organ involvement (except central nervous system) died in ICU and only $20 \%$ survived. Two patients who survived had only 
three organs involved on admission to ICU and four or more organs were affected in patients who died. This indicates the importance of seeking critical care or critical care outreach management in leptospirosis patients with the involvement of three organs.

CNS involvement was found in six patients and all had six organs involved. All six patients died in the ICU. According to this case series, involvement of five or more organs were associated with increased mortality. Furthermore, a combination of cardiac, respiratory and CNS involvement was associated with increased mortality.

Diagnosis of leptospirosis was based on symptomatology and investigations including the presence of antibodies but not on detecting spirochetes in body fluids in this case series. Four patients died within the first week of their illness and leptospira antibodies were negative in those cases.

\section{References}

1. Palaniappan RU, Ramanujam S, Chang YF. Leptospirosis: pathogenesis, immunity and diagnosis. Current Opinion in Infectious Disease. 2007;20(3):284-292.

2. Green-Mckenzie J, Shoff WH. Leptospirosis in Human. E Medicine. 2008(WebMD).

3. Carvalho CR, Bethlem EP. Pulmonary complications of leptospirosis. Clinical Chest Medicine 2002;23(2):469-478.

4. Im JG, Yeon KM, Han MC, Kim CW, Webb WR and et al. Leptospirosis of the lung: radiographic finding in 58 patients. American Journal of Roentgenol 1989;152(5):955-959.

$* * * * * * * * * * * * * * * * * * * * * * * * * * * * * * * * * * * * * * * * * * * * * * * * * * * * * * * * * * * * * * * * * * * * * * * * * *$

\section{THE COLLEGE OF ANAESTHESIOLOGISTS OF SRI LANKA}

\section{FREE PAPERS :}

The rules of the competition are:

1. Open to all medical practitioners in Anaesthesia.

2. Duration of the presentation must be no longer than 15 minutes.

3. The work in whole or in part should not have been presented or published elsewhere.

4. An abstract of not more than 200 words should be submitted before the closing date.

5. The first right of publication of the entries submitted lies with the editor of the College of Aanesthesiologists of Sri Lanka.

6. The decision of the panel of judges in all matters relating to the competition including questions relating to eligibility shall be final.

7. The prize will not be awarded if the Judges decide the presentation is not up to standard.

The closing date for the 2012 competition is $31^{\text {st }}$ October 2012. The abstract should reach the undersigned before the closing date. Any expenditure incurred in the preparation of the slides for papers that were selected for presentation will be reimbursed by the College. 3 copies of the full text should be submitted on or before $31^{\text {st }}$ October 2012 to the anaesthetic office. 